\title{
Ultrasonido para la evaluación de la deglución: una revisión narrativa
}

\author{
Ultrasound for swallowing assessment: Narrative \\ review
}

Jorge Sepúlveda

Hospital San Luis de Buin-Paine

IPChile

Roberto Rojas

Universidad Autónoma de Chile Universidad de Las Américas

\section{Michelle Casanova}

Universidad Mayor Departamento de NeurologíaNeurocirugía, Hospital Clínico Universidad de Chile

\section{Contacto con el autor: Roberto Rojas Santiago - Chile Correo-e: roberto.rojas.segovia@gmail.com}

\section{RESUMEN}

El presente estudio explora evidencia sobre la evaluación de la deglución, usando la técnica de ultrasonido. En relación a la estrategia búsqueda, la revisión se desarrolló en PubMed y Scielo, utilizando una adaptación de las recomendaciones del manual Cochrane para revisiones sistemáticas. Se seleccionaron los términos "Deglutition" "Swallowing", "Ultrasonography" y "Ultrasound" en inglés y en español. Se incluyeron inicialmente estudios originales publicados entre enero del 2000 y enero del 2019. De acuerdo con los criterios de elegibilidad, se incluyeron 24 artículos originales sobre el uso de ultrasonido para evaluar algún aspecto de la deglución en las etapas oral y faríngeas. Los artículos seleccionados ofrecen datos cuantitativos de parámetros e indicadores de la deglución. A partir de estos estudios, se recogió información cualitativa y cuantitativa sobre la movilidad lingual, la elevación laríngea, la movilidad faríngea lateral, la movilidad del hioides, la movilidad del músculo genihioideo, la apertura del esfínter esofágico superior, el cierre glótico, además de estudios sobre la posibilidad de detectar episodios de penetración laríngea y aspiración. Los resultados sugieren que existe evidencia positiva sobre la utilidad del ultrasonido para evaluar la deglución, sin embargo, se requiere de más estudios que analicen la eficiencia diagnóstica en población con disfagia.

Palabras Clave: deglución, ultrasonido, ecografía.

\section{ABSTRACT}

The present study explores evidence on swallowing assessment using ultrasound technique. Regarding the search strategy, the review was carried out in PubMed and Scielo, using an adaptation of the recommendations from the Cochrane manual for systematic reviews. The terms "Deglutition" "Swallowing", "Ultrasonography" and "Ultrasound" were selected in English and Spanish. Original studies published between January 2000 and January 2019 were initially selected. According to the elegibility criteria, 24 original studies dealing with the use of ultrasound to evaluate some aspect of swallowing in both oral and pharyngeal stages. Selected articles offer quantitative data on swallowing parameters and indicators. From these studies, qualitative and quantitative information on lingual mobility, laryngeal elevation, lateral pharyngeal mobility, hyoid mobility, genihyoid muscle mobility, upper esophageal sphincter opening, glotic closure was collected, in addition to studies on the possibility of detecting episodes of laryngeal penetration and aspiration. The results suggest that there is positive evidence on the usefulness of ultrasound to assess swallowing, however, more studies are required to analyze diagnostic efficiency in a population with dysphagia.

Keywords: deglutition, ultrasonography, ultrasound. 


\section{Introducción}

Deglución se define como una compleja actividad neuromuscular producto de una serie de movimientos coordinados de distintos grupos musculares de la cavidad oral, faringe y esófago, cuyo fin es permitir que los alimentos sean transportados desde la boca al estómago. Para contenidos líquidos, el proceso de deglución se divide en: etapa preparatoria oral, etapa oral, etapa faríngea y etapa esofágica. Para alimentos sólidos se describe el modelo de proceso de alimentación, en el que la etapa oral se subdivide en: Etapa I de transporte, etapa de procesamiento de alimentos, y en etapa II de transporte.

En ambas clasificaciones, tanto para la etapa preparatoria como para la etapa oral, la movilidad lingual es crucial para su correcto funcionamiento. Para la etapa faríngea, en cambio, se describen acciones neuromusculares como el movimiento de las cuerdas vocales para sellar la glotis, el movimiento de los aritenoides, el desplazamiento del hueso hioides y de la laringe, y la apertura del esfínter esofágico superior (EES) (Matsuo \& Palmer, 2008).

El diagnóstico de los trastornos deglutorios o disfagia, tradicionalmente se realiza a través de dos métodos: La evaluación clínica y la evaluación instrumental. La evaluación clínica brinda al profesional de la salud la oportunidad de observar la función general del proceso deglutorio de un individuo en un contexto determinado (Cichero \& Murdoch, 2006). No obstante, en ocasiones es necesario realizar una evaluación instrumental para detectar la presencia de algunos signos y complicaciones de la disfagia, como la aspiración de bolo alimenticio (presencia de bolo en la vía respiratoria por debajo de la glotis). La exploración con técnicas de imagen proporciona al clínico detalles precisos sobre las estructuras anatómicas involucradas en la deglución y permite identificar con seguridad los signos de trastorno deglutorio (Cichero \& Murdoch, 2006).

En el contexto de la evaluación instrumental, las técnicas más utilizadas son la Videofluoroscopía (VFC) y la Evaluación Nasofibroscópica de la deglución (FEES). Existe una amplia gama de estudios de validez de contingencia y eficiencia diagnóstica que ubican a estas dos técnicas de imagen como los estándares en términos de examen de confirmación diagnóstica de disfagia. Sin embargo, si bien ambos procedimientos proporcionan imágenes dinámicas de la biomecánica de la deglución, a medida que aumenta la comprensión de la deglución se reconocen ciertas limitaciones de estas pruebas en base a la necesidad de proporcionar información no solo cualitativa del proceso de deglución, sino que también cuantitativa (Huckabee, Macrae, \& Lamvik, 2015).

Otro procedimiento instrumental para valorar el proceso deglutorio es el Ultrasonido (US). EL US es una técnica que visualiza la cavidad oral y la hipofaringe durante la deglución, utilizando un transductor ubicado en la región submentoniana para obtener la imagen (Cichero \& Murdoch, 2006). Dicho procedimiento no se utiliza con frecuencia en el proceso diagnóstico de la disfagia ni en la exploración del proceso deglutorio en su fase orofaríngea, ya que existe literatura que señala la dificultad de la técnica para detectar episodios de aspiración (Logemann, 1998). No obstante, se ha demostrado que esta técnica ha sido útil para realizar observaciones 
cualitativas de diferentes estructuras participantes en el proceso deglutorio. Además, las innovaciones metodológicas de las últimas décadas han permitido llevar al US a realizar valoraciones cuantitativas de diversos aspectos dinámicos de la deglución en diferentes modalidades de imagen (Chi-Fishman, 2005). Se ha descrito que incluso el US puede entregar al clínico información en tiempo real tanto para diagnosticar como para verificar los efectos que las estrategias terapéuticas tienen en la fisiología deglutoria.

Estas últimas evidencias nos llevan a plantear nuestra pregunta de investigación: ¿EI US es útil como evaluación instrumental de la deglución? Para responderla nos planteamos el objetivo de explorar el estado del arte de la evaluación de la deglución con el uso de la técnica de US a través de una revisión narrativa de la literatura. Con la evidencia recabada pretendemos discutir sobre el US como una técnica cualitativa y cuantitativa para evaluar la biomecánica deglutoria.

\section{Metodología}

Para realizar la búsqueda de evidencia en esta revisión narrativa, se utilizó una adaptación de las recomendaciones sugeridas por el Manual Cochrane para revisiones sistemáticas de intervenciones (Higgins \& Green, 2011).
Dos revisores seleccionaron los estudios utilizando un protocolo validado por un fonoaudiólogo experto en deglución (Tabla $n^{\circ} 1$ ). Los desacuerdos entre los revisores fueron resueltos mediante consenso aplicando la pauta de validación de artículos (Tabla $n^{\circ} 2$ ). La búsqueda se realizó en junio del 2019 en las bases de datos PubMed y Scielo. La primera búsqueda utilizó en la operación 1 el término clave MeSH "Ultrasonography", más el boleano de intersección "AND" que antecedía a la operación 2 con el término clave MeSH "Deglutition". Una segunda búsqueda se realizó con el término libre "Ultrasound" en la primera operación, el booleano de intersección "AND" y el término "Swallowing" en la segunda operación. Estos últimos términos abundan en la literatura, aunque no son considerados oficialmente palabras claves MeSH. El mismo procedimiento de búsqueda se efectuó con los equivalentes en español (Ultrasonografía, Ultrasonido y Deglución). Las búsquedas se realizaron de la misma manera tanto en Pubmed como en Scielo, e incluyeron resultados para los apartados: título y abstract. Los filtros de elegibilidad fueron: a) fecha de publicación (que abarcó el periodo entre enero del 2000 a enero del 2019); b) idioma (inglés y español) y c) tipo de estudio (originales con diseños del tipo ensayos clínicos observacionales, ensayos clínicos de eficiencia diagnóstica, ensayos clínicos de validez de prueba). 
Tabla 1

Protocolo de búsqueda.

№ Indicaciones de búsqueda y selección para revisores

1 El revisor buscará artículos en Scielo y PubMed con la siguiente combinación de términos y operadores boleanos para Titulo y Abstract: A) Operación 1.1: Ultrasonography; Boleano de intersección: AND; Operación 2.1: Deglutition. B) La segunda búsqueda posee el Operador 1.2: Ultrasound; Boleano de intersección AND; operador 2.2: Swallowing. El revisor buscará luego incorporando los términos equivalentes en español (Ultrasonografía, Ultrasonido y Deglución).

2 Guardará las búsquedas realizadas para lo cual debe crear un perfil en las bases de datos exploradas (Scielo y PubMed).

3 El revisor aplicará los filtros definidos para la elegibilidad de los artículos.

4 El revisor listará en planilla Excel los siguientes antecedentes de los artículos encontrados con los criterios de búsqueda: Nombre original artículo / Año de publicación / Autor / Objetivo del estudio / Criterio de Exclusión (CE) / Estado de verificación.

5 El revisor analizará el título y el abstract del artículo identificando el tipo de CE cuando se estime la exclusión.

6 Con los artículos resultantes se procede a la validación de los que finalmente se incorporarán al análisis (Tabla $\left.n^{\circ} 2\right)$.

7 Los artículos que cumplen elegibilidad para la revisión son aquellos que poseen la totalidad de las preguntas de la pauta de validación con respuesta "SI".

Tabla 2

Pauta de validación de artículos utilizada en caso de desacuerdo entre los revisores.

a) ¿Los objetivos de la investigación están claramente especificados en el abstract o en la introducción?

b) ¿Se describe claramente la técnica de ultrasonido utilizada?

c) ¿Las medidas utilizadas para cuantificar aspectos de la deglución están claramente determinadas?

d) ¿La población del estudio está claramente descrita?

e) ¿El artículo describe sus limitaciones?

f) ¿Las conclusiones se relacionan con los objetivos planteados en la investigación?

Tabla 3

Criterios de Exclusión (CE) utilizados para sancionar en la búsqueda.

Criterios de Exclusión para sancionar.

CE 1 El artículo no trata sobre el uso de ultrasonido para evaluar algún aspecto de la deglución.

CE 2 El artículo no trata sobre las etapas oral y faríngeas de la deglución.

CE 3 El artículo no analiza cuantitativamente parámetros e indicadores de la deglución. 


\section{Resultados y discusión}

Esta sección se organiza en dos apartados: a) resultados referidos a la cantidad y a las características de los artículos seleccionados y b) resultados relacionados con la información que se obtuvo del análisis de los artículos.

\section{a) Cantidad y características de los artículos seleccionados.}

Con los términos de búsqueda establecidos se hallaron 5230 artículos, luego de ser eliminados los duplicados. Con los filtros declarados se excluyen por fecha de publicación 1941 artículos; por lenguaje o idioma de publicación 576 investigaciones; y finalmente por tipo de estudio 2681 trabajos no fueron considerados en esta revisión. Con todo lo anterior, 32 artículos fueron seleccionados para ser leídos siguiendo el protocolo de elegibilidad. Se excluyeron 8 artículos que cabían dentro de los criterios de exclusión determinados, cuyo detalle se muestra en la Tabla $n^{\circ} 4$. Finalmente, se consideraron 24 artículos para esta revisión. La Figura 1 contiene el flujograma elaborado para llevar a cabo la revisión, donde se contemplan todas las etapas de la selección de artículos. Cabe destacar que las búsquedas en Scielo no arrojaron artículos elegibles con los criterios planteados para esta revisión.

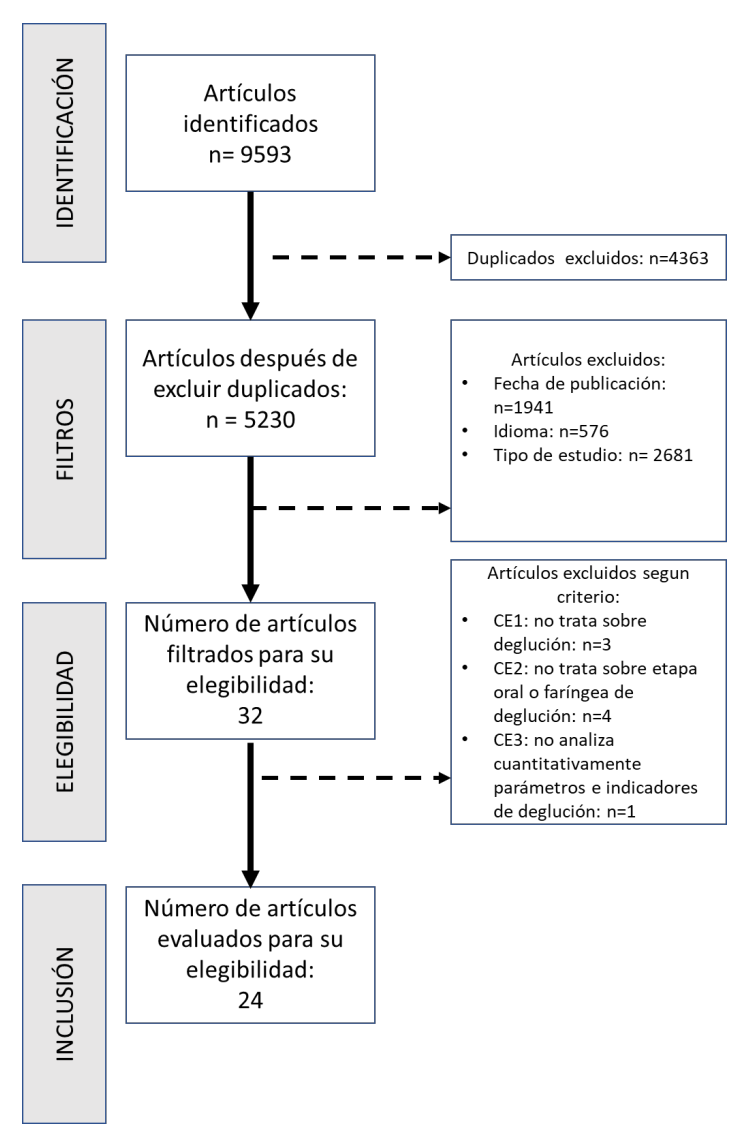

Figura 1. Flujo de selección y elegibilidad de los artículos. 
Tabla 4

Detalle de cantidad de artículos excluidos en cuanto a motivo.

\begin{tabular}{lc}
\hline Criterio de exclusión (CE) & $\mathrm{n}$ \\
\hline CE 1 El artículo no trata sobre el uso de ultrasonido para evaluar algún aspecto de la deglución. & 3 \\
CE 2 El artículo no trata sobre las etapas oral y faríngeas de la deglución. & 4 \\
CE 3 El artículo no analiza cuantitativamente parámetros e indicadores de la deglución. & 1 \\
Total excluidos & -8 \\
\hline
\end{tabular}

\section{Resultados y discusión}

Todos los artículos seleccionados e incluidos en la revisión corresponden a estudios en inglés. De los 24 artículos, 2 analizan el proceso de deglución como una función completa valorando más de una acción neuromuscular a través de US. En los 22 artículos restantes el análisis efectuado a través del US se relacionaba a una etapa en particular, ya sea faríngea (16 artículos), preparatoria u oral (4 artículos para estas últimas etapas). También se encontraron 2 artículos que estudian con US la etapa faríngea y oral de forma unificada. Entre los artículos que cumplen los criterios de selección existen se encuentran varios relacionados directamente con disfagia; esto se declara en este apartado toda vez que el objetivo de esta revisión es demostrar la utilidad en la evaluación de la deglución, independiente de la presencia o no de alguna alteración.
En relación con el tipo de muestra utilizada, nueve estudios incluyen únicamente a población sana, seis a población con disfagia, y nueve estudios compararon e incluyen a población con disfagia y a sujetos sanos. Todos los artículos incorporan mujeres y hombres en sus muestras. Mayores detalles demográficos y técnicos de los estudios se anexan en la Tabla $n^{\circ} 5$.

En la Tabla $n^{\circ} 5$ se destaca la gran heterogeneidad tanto de equipos utilizados como de técnicas y procedimientos. La mayoría de los estudios si bien presenta gran detalle de las marcas y modelos de los equipos utilizados, no especifica las configuraciones del software ni los medios ecogénicos utilizados (por ejemplo, si utilizaron presets para partes blandas, para sistema nervioso, para cavidades, o el tipo de gel utilizado). Lo anterior dificulta la presentación más estandarizada de los resultados. También, obstaculiza la comparación más precisa de los hallazgos que los investigadores de esos estudios exponen. 
Tabla 5

Resumen de los artículos analizados mencionado metodología y aspectos técnicos utilizados en la investigación.

\begin{tabular}{|c|c|c|c|c|}
\hline Autor & Título & Muestra & Especificaciones técnicas & Procedimiento realizado \\
\hline $\begin{array}{l}\text { Ahn et al. } \\
(2015)\end{array}$ & $\begin{array}{l}\text { Reliability of Ultrasound } \\
\text { Evaluation of Hyoid- } \\
\text { Larynx Approximation } \\
\text { with Positional Change. }\end{array}$ & $\begin{array}{l}\mathrm{n}=20 \\
\text { Características: } \\
\text { Sujetos sanos. } \\
\text { Promedio: Rango } \\
\text { entre 20-40 años. }\end{array}$ & $\begin{array}{l}\text { Equipo utilizado: Transductor } \\
\text { lineal de } 7.5 \mathrm{MHz} \text { (Phillips } \\
\text { Envisor HD, EE. UU.). } \\
\text { Ubicación transductor: El } \\
\text { transductor se colocó a lo } \\
\text { largo del eje longitudinal en la } \\
\text { línea media anterior del } \\
\text { cuello. } \\
\text { Gel utilizado: No informa. }\end{array}$ & $\begin{array}{l}\text { Se evalúa en posición sentada y en } \\
\text { decúbito. En la posición sentada, se le } \\
\text { pidió al paciente que se siente } \\
\text { cómodamente con la cabeza } \\
\text { apoyándose ligeramente contra una } \\
\text { pared y el cuello en posición neutral. } \\
\text { En posición supina, se le pidió al } \\
\text { paciente que trague } 5 \text { ml de líquido y } \\
\text { mantenga la cabeza ligeramente } \\
\text { elevada. Se midió la distancia hioides - } \\
\text { laringe en reposo y en deglución. } \\
\text { También se valoró la elevación } \\
\text { laríngea. }\end{array}$ \\
\hline $\begin{array}{l}\text { Cheng, } \\
\text { Lee, Ahuja, } \\
\text { \& Tong } \\
\text { (2018) }\end{array}$ & $\begin{array}{l}\text { Sonographic assessment } \\
\text { of swallowing in } \\
\text { irradiated } \\
\text { nasopharyngeal } \\
\text { carcinoma patients. }\end{array}$ & $\begin{array}{l}\mathrm{n}=40 \\
\text { Características: } \\
\text { Promedio de } 9,7 \\
\text { años desde la } \\
\text { finalización del } \\
\text { tratamiento. Todos } \\
\text { presentaban disfagia. } \\
\text { Promedio: } 53,9\end{array}$ & $\begin{array}{l}\text { Equipo utilizado: Sistema de } \\
\text { ultrasonido portátil Mindray } \\
\text { M7 (Mindray, Shenzhen, } \\
\text { China) y un transductor lineal } \\
\text { de } 6 \text { a } 14 \mathrm{MHz} \text {. } \\
\text { Ubicación transductor: Se } \\
\text { ubicó en la región } \\
\text { submentoniana en } \\
\text { orientación coronal justo } \\
\text { encima del hueso hioides. } \\
\text { Gel utilizado: Gel conductor. }\end{array}$ & $\begin{array}{l}\text { Cada participante recibió instrucciones } \\
\text { de sentarse erguido, relajado y } \\
\text { mantener la cabeza en una posición } \\
\text { neutral durante todo el examen. Luego } \\
\text { se le indicó al participante que } \\
\text { deglutiera } 5 \mathrm{ml} \text { de agua a temperatura } \\
\text { ambiente en una cuchara estándar. A } \\
\text { lo largo de la prueba de deglución, la } \\
\text { posición del transductor se ajustó } \\
\text { anteroposteriormente para seguir el } \\
\text { movimiento del hueso hioides y se } \\
\text { mantuvo justo por encima del hueso } \\
\text { hioides. La prueba se repitió dos veces } \\
\text { para obtener un conjunto de tres } \\
\text { mediciones. Además, se realizó VFC } \\
\text { para obtener otra serie de mediciones } \\
\text { fisiológicas. }\end{array}$ \\
\hline $\begin{array}{l}\text { Feng et al. } \\
(2015)\end{array}$ & $\begin{array}{l}\text { Ultrasonographic } \\
\text { evaluation of geniohyoid } \\
\text { muscle and hyoid bone } \\
\text { during swallowing in } \\
\text { young adults. }\end{array}$ & $\begin{array}{l}\mathrm{n}=40 \\
\text { Características: } \\
\text { Sujetos sanos. } \\
\text { Promedio: Rango } \\
\text { entre 20-40 años. }\end{array}$ & $\begin{array}{l}\text { Equipo utilizado: Biosound } \\
\text { MyLab25 (Grupo Esaote, } \\
\text { Génova, Italia), con un } \\
\text { transductor de matriz lineal } \\
\text { de } 18 \mathrm{MHz} \text {. } \\
\text { Ubicación transductor: Para } \\
\text { valorar al M. Genihioideo el } \\
\text { transductor se colocó en la } \\
\text { región medial submentoniana } \\
\text { longitudinalmente, siendo } \\
\text { realizada en modo M (modo } \\
\text { movimiento). Para la } \\
\text { valoración de la movilidad del } \\
\text { hueso hioides el transductor } \\
\text { se colocó un poco más } \\
\text { posterior a la posición } \\
\text { descrita anteriormente. } \\
\text { Gel utilizado: No informa }\end{array}$ & $\begin{array}{l}\text { Se evalúa en posición sentada, supino, } \\
\text { decúbito lateral derecho. El } \\
\text { participante recibió instrucciones de } \\
\text { tomar } 10 \mathrm{ml} \text { de agua mineral a través } \\
\text { de una bombilla para evitar el } \\
\text { movimiento de la cabeza, en la que } \\
\text { debía mantener el líquido en la boca } \\
\text { hasta esperar la instrucción de } \\
\text { deglutir. } \\
\text { El probador sostuvo la barbilla para } \\
\text { evitar el movimiento de la cabeza } \\
\text { durante la deglución. Esta prueba se } \\
\text { repitió cinco veces, con un intervalo de } \\
40 \text { segundos de intervalo entre } \\
\text { degluciones. }\end{array}$ \\
\hline $\begin{array}{l}\text { Hsiao, } \\
\text { Chang, } \\
\text { Chen, }\end{array}$ & $\begin{array}{l}\text { Application of } \\
\text { Ultrasonography in } \\
\text { Assessing }\end{array}$ & $\begin{array}{l}\mathrm{n}=100 \\
\text { Características: } 10 \\
\text { sujetos sanos; } 60\end{array}$ & $\begin{array}{l}\text { Equipo utilizado: Transductor } \\
\text { curvilíneo (BS3C673 Convex } \\
\text { Array, 3.5MHz, BSUS20-32C; }\end{array}$ & $\begin{array}{l}\text { Cada participante deglutió } 5 \mathrm{ml} \text { de } \\
\text { agua por cada intento en posición } \\
\text { sedente. Las imágenes grabadas fueron }\end{array}$ \\
\hline
\end{tabular}




\begin{tabular}{|c|c|c|c|c|}
\hline Autor & Título & Muestra & Especificaciones técnicas & Procedimiento realizado \\
\hline $\begin{array}{l}\text { Chang, \& } \\
\text { Wang } \\
(2012)\end{array}$ & $\begin{array}{l}\text { Oropharyngeal } \\
\text { Dysphagia in Stroke } \\
\text { Patients. }\end{array}$ & $\begin{array}{l}\text { sujetos con ACV ( } 41 \\
\text { isquémico, } 19 \\
\text { hemorrágico), } 30 \\
\text { presentaban } \\
\text { alimentación por } \\
\text { sonda y } 30 \text { tenían } \\
\text { alimentación por vía } \\
\text { oral. } \\
\text { Promedio: } 57 \text { años } \pm \\
38 \text { días. }\end{array}$ & $\begin{array}{l}\text { Broadsound Corporation, } \\
\text { Taiwán). } \\
\text { Ubicación transductor: Se } \\
\text { ubicó en el plano sagital del } \\
\text { área submentoniana. } \\
\text { Gel utilizado: No informa }\end{array}$ & $\begin{array}{l}\text { analizadas en cuadros individuales para } \\
\text { determinar el cambio máximo en el } \\
\text { espesor de la lengua y el } \\
\text { desplazamiento del hueso hioides. } \\
\text { Además, } 12 \text { pacientes con ACV se les } \\
\text { realizó VFC para complementar los } \\
\text { resultados. }\end{array}$ \\
\hline $\begin{array}{l}\text { Huang, } \\
\text { Hsieh, } \\
\text { Chang, } \\
\text { Chen, \& } \\
\text { Wang } \\
\text { (2009) }\end{array}$ & $\begin{array}{l}\text { Ultrasonographic } \\
\text { Evaluation of Hyoid- } \\
\text { Larynx Approximation in } \\
\text { Dysphagic Stroke } \\
\text { Patients. }\end{array}$ & $\begin{array}{l}\mathrm{n}=55 \\
\text { Características: } 40 \\
\text { usuarios } \\
\text { diagnosticados con } \\
\text { ACV ( } 20 \text { con disfagia, } \\
\text { y } 20 \text { sin disfagia), y } \\
15 \text { sujetos sin } \\
\text { patología de base. } \\
\text { Promedio: } 61.2 \\
\pm 16.4 \text {. }\end{array}$ & $\begin{array}{l}\text { Equipo utilizado: Ultrasonido } \\
\text { portátil (Sistema de } \\
\text { ultrasonido T3000, Terason, } \\
\text { Burlington, MA, EE. UU.), con } \\
\text { transductor lineal de } 10 \mathrm{MHz} \\
\text { (modelo 5C2). } \\
\text { Ubicación transductor: Fue } \\
\text { colocado a lo largo el eje } \\
\text { longitudinal en la línea media } \\
\text { del cuello. } \\
\text { Gel utilizado: No informa. }\end{array}$ & $\begin{array}{l}\text { Los } 55 \text { pacientes fueron clasificados en } \\
3 \text { grupos utilizando la escala de } \\
\text { penetración - aspiración (PAS), (grupo } \\
1 \text { PAS 1; grupo dos, penetración, PAS: } \\
\text { 2-5; grupo 3, aspiración; PAS 6-8). Los } \\
\text { grupos 1, 2, } 3 \text { tenían } 21,20 \text { y } 11 \\
\text { pacientes, la mayoría por ACV. Se les } \\
\text { pidió deglutir tres veces con un } \\
\text { intervalo de 1-2 min entre cada } \\
\text { deglución. Se les administró agua (de } 2 \\
\text { a } \\
3 \text { ml) en cada deglución para facilitar el } \\
\text { proceso. } \\
\text { Se definió el porcentaje de cambio de } \\
\text { la aproximación hioides-laringe como } \\
\text { el valor obtenido al dividir la distancia } \\
\text { de aproximación por la distancia de } \\
\text { reposo, expresada como un } \\
\text { porcentaje. }\end{array}$ \\
\hline $\begin{array}{l}\text { Jadcherla } \\
\text { et al. } \\
\text { (2006) }\end{array}$ & $\begin{array}{l}\text { Correlation of glottal } \\
\text { closure using concurrent } \\
\text { ultrasonography and } \\
\text { nasolaryngoscopy in } \\
\text { children: a novel } \\
\text { approach to evaluate } \\
\text { glottal status. }\end{array}$ & $\begin{array}{l}\mathrm{n}=10 \\
\text { Características: } 2 \\
\text { fueron evaluados por } \\
\text { disfagia, } 2 \text { por } \\
\text { ronquera, } 3 \text { por } \\
\text { laringomalacia y } 3 \\
\text { por estridor. } \\
\text { Además, } 5 \text { de los ya } \\
\text { mencionando tenían } \\
\text { un diagnóstico } \\
\text { adicional de RGE } \\
\text { Promedio: } 4.5 \text { meses } \\
\text { a } 7.1 \text { años, (media = } \\
2.8 \text { años, mediana = } \\
2 \text { años). }\end{array}$ & $\begin{array}{l}\text { Equipo utilizado: Se utilizaron } \\
\text { los equipos Acuson Sequoia } \\
512 \text { (Siemens, Mountain } \\
\text { View, CA) equipado con un } \\
15 \mathrm{~L} 8(7,1-14,0 \mathrm{MHz} \text { ) con } \\
\text { transductor lineal y un Philips } \\
\text { HDI } 5000 \text { (Bothell, WA) con } \\
\text { una matriz lineal de 12-5 } \\
\text { MHz. Ambos son } \\
\text { transductores lineales de } \\
\text { banda ancha de alta } \\
\text { frecuencia. } \\
\text { Ubicación transductor: El } \\
\text { transductor se colocó en la } \\
\text { parte anterior del cuello a } \\
\text { nivel de pliegues vocales. } \\
\text { Gel utilizado: Se menciona la } \\
\text { utilización de gel como medio } \\
\text { de acoplamiento. No se } \\
\text { especifican más detalles. }\end{array}$ & $\begin{array}{l}\text { La realización del ultrasonido fue en } \\
\text { paralelo con la realización de una FEES, } \\
\text { en donde el cierre cordal fue } \\
\text { reconocido durante la deglución o la } \\
\text { fonación. Dicho procedimiento fue } \\
\text { analizado por dos investigadores que } \\
\text { estaban cegados a las imágenes de } \\
\text { FEES. El cierre cordal fue reconocido } \\
\text { durante la fonación tras la emisión de } \\
\text { sonidos vocálicos (aah - eeh y llanto), } \\
\text { mientras que para deglución no se } \\
\text { especifica la instrucción o método } \\
\text { aplicado. }\end{array}$ \\
\hline $\begin{array}{l}\text { Kim \& Kim } \\
(2012)\end{array}$ & $\begin{array}{l}\text { Lateral Pharyngeal Wall } \\
\text { Motion Analysis Using } \\
\text { Ultrasonography in } \\
\text { Stroke Patients with }\end{array}$ & $\begin{array}{l}\mathrm{n}=41 \\
\text { Características: } \\
\text { Usuarios con ACV, } \\
\text { todos presentabas }\end{array}$ & $\begin{array}{l}\text { Equipo utilizado: Sistema de } \\
\text { ultrasonido ACUSON Antares, } \\
\text { edición premium; Siemens } \\
\text { Medical Solutions, Mountain }\end{array}$ & $\begin{array}{l}\text { La evaluación ecográfica fue realizada } \\
\text { por el mismo operador para todos los } \\
\text { pacientes. Todos estaban sentados en } \\
\text { una posición cómoda con la cabeza en }\end{array}$ \\
\hline
\end{tabular}




\begin{tabular}{|c|c|c|c|c|}
\hline Autor & Título & Muestra & Especificaciones técnicas & Procedimiento realizado \\
\hline & Dysphagia. & $\begin{array}{l}\text { disfagia. } \\
\text { Promedio: } 60.9, \pm \\
13.6 \text { años. }\end{array}$ & $\begin{array}{l}\text { View, CA, EE. UU. Transductor } \\
\text { de matriz convexa electrónica } \\
\text { de 5,71 MHz (Modelo CH 6-2), } \\
\text { con pantalla dividida en modo } \\
\text { B (arriba) y modo M (abajo). } \\
\text { Ubicación transductor: } \\
\text { Verticalmente a cada lado del } \\
\text { cuello, con el borde superior } \\
\text { aproximadamente } 1 \text { cm por } \\
\text { debajo del canal auditivo } \\
\text { externo. } \\
\text { Gel utilizado: Gel de } \\
\text { acoplamiento para eliminar } \\
\text { cualquier interfaz aérea entre } \\
\text { la piel y el transductor. }\end{array}$ & $\begin{array}{l}\text { una posición neutral, y se usó un } \\
\text { dispositivo de soporte para reducir los } \\
\text { movimientos movimiento durante la } \\
\text { deglución. Los participantes se } \\
\text { agruparon en grupos: Grupo A; } \\
\text { usuarios con disfagia que presentaban } \\
\text { penetración o aspiración, y grupo B, } \\
\text { usuarios con disfagia que no } \\
\text { presentaban episodios de penetración } \\
\text { ni aspiración, más los participantes del } \\
\text { grupo control. Se realizó VFC en } \\
\text { simultáneo con US. Se evaluó } \\
\text { movimiento (desplazamiento) y } \\
\text { duración del movimiento de la pared } \\
\text { faríngea lateral. A cada participante se } \\
\text { le administró } 5 \text { ml de agua que tenía } \\
\text { que "sostener" en la boca, a la espera } \\
\text { de la indicación de deglutir por parte } \\
\text { del examinador. }\end{array}$ \\
\hline $\begin{array}{l}\text { Komori, } \\
\text { Hyodo, \& } \\
\text { Gyo (2008) }\end{array}$ & $\begin{array}{l}\text { A Swallowing Evaluation } \\
\text { with Simultaneous } \\
\text { Videoendoscopy, } \\
\text { Ultrasonography and } \\
\text { Videofluorography in } \\
\text { Healthy Controls. }\end{array}$ & $\begin{array}{l}\mathrm{n}=8 \\
\text { Características: } \\
\text { Voluntarios sanos. } \\
\text { Promedio: } 29,6 \text { (25- } \\
30 \text { años). }\end{array}$ & $\begin{array}{l}\text { Equipo utilizado: Ultrasonido } \\
\text { con sistema de tomografía } \\
\text { (SSD-5000, Aloka Co. Ltd., } \\
\text { Tokio, Japón). } \\
\text { Ubicación transductor: Se } \\
\text { colocó un transductor lineal } \\
\text { electrónico de 7,5 Hz } \\
\text { verticalmente en el costado } \\
\text { del cuello. } \\
\text { Gel utilizado: No informa. }\end{array}$ & $\begin{array}{l}\text { Cada sujeto se posicionó en la posición } \\
\text { en la que normalmente come. Se } \\
\text { realizó de forma simultánea FEES, VFC } \\
\text { y US, y se les solicitó deglutir } 15 \mathrm{ml} \text { de } \\
\text { bario ( } 145 \% \mathrm{w} / \mathrm{v}) \text {. Cada sujeto repitió } \\
\text { el procedimiento de deglución } 3 \text { veces. }\end{array}$ \\
\hline $\begin{array}{l}\text { Kuhl, Eicke, } \\
\text { Dieterich, } \\
\text { \& Urban } \\
(2003)\end{array}$ & $\begin{array}{l}\text { Sonographic analysis of } \\
\text { laryngeal elevation } \\
\text { during swallowing. }\end{array}$ & $\begin{array}{l}\mathrm{n}=60 \\
\text { Características: } 42 \\
\text { sujetos sanos; } 18 \\
\text { pacientes con } \\
\text { disfagia neurogénica. } \\
\text { Promedio: Sujetos } \\
\text { sanos } 57 \pm 19 \text { años, } \\
\text { sujetos con disfagia } \\
63 \pm 8 \text { años. }\end{array}$ & $\begin{array}{l}\text { Equipo utilizado: Transductor } \\
\text { lineal de } 7,5 \mathrm{~Hz} \text {. (Philips SD } \\
800 \text {, Irvine CA). } \\
\text { Ubicación transductor: Se } \\
\text { ubicó en una posición } \\
\text { longitudinal por encima de la } \\
\text { laringe. } \\
\text { Gel utilizado: No informa. }\end{array}$ & $\begin{array}{l}\text { Los sujetos se colocaron en posición } \\
\text { supina con la cabeza ligeramente } \\
\text { elevada. Ambos grupos de } \\
\text { participantes fueron evaluados a través } \\
\text { de US con } 5 \text { y } 10 \text { ml de líquido. Todos } \\
\text { los ensayos de deglución se realizaron } \\
\text { seis veces, en el que las medidas } \\
\text { posteriormente fueron promediadas } \\
\text { para su cálculo. La imagen con la } \\
\text { mayor y menor distancia entre el } \\
\text { hueso hioides y el cartílago tiroideo } \\
\text { fueron identificados por función cine- } \\
\text { loop. }\end{array}$ \\
\hline $\begin{array}{l}\text { Kwak et al. } \\
\text { (2018) }\end{array}$ & $\begin{array}{l}\text { Influence of Nasogastric } \\
\text { Tubes on Swallowing in } \\
\text { Stroke Patients: } \\
\text { Measuring Hyoid Bone } \\
\text { Movement With } \\
\text { Ultrasonography. }\end{array}$ & $\begin{array}{l}\mathrm{n}=70 \\
\text { Características: } 45 \\
\text { participantes con } \\
\text { ACV (Grupo A } 20 \\
\text { sujetos con SNG, } \\
\text { Grupo B, } 25 \text { sujetos } \\
\text { sin SNG (25), y } 25 \\
\text { adultos sanos sin } \\
\text { disfagia ni } \\
\text { antecedente de ACV } \\
\text { (Grupo C). }\end{array}$ & $\begin{array}{l}\text { Equipo utilizado: Accuvix V10, } \\
\text { Medison, con un transductor } \\
\text { de matriz curva de 3-6 MHz. } \\
\text { Ubicación transductor: Se } \\
\text { ubicó el transductor en el } \\
\text { plano medio sagital, inferior al } \\
\text { área mental. } \\
\text { Gel utilizado: No informa }\end{array}$ & $\begin{array}{l}\text { Se les pidió a los participantes que } \\
\text { deglutieran } 1 \mathrm{ml} \text { de agua, mientras } \\
\text { todo el proceso de deglución era } \\
\text { monitoreado y grabado en video por } \\
\text { US. Los participantes del grupo A } \\
\text { fueron examinados dos veces: una vez } \\
\text { con SNG (grupo A1) y una vez después } \\
\text { de eliminar la SNG (grupo A2). La } \\
\text { prueba se repitió tres veces a } \\
\text { intervalos de un minuto para cada } \\
\text { participante. A los participantes del }\end{array}$ \\
\hline
\end{tabular}




\begin{tabular}{|c|c|c|c|c|}
\hline Autor & Título & Muestra & Especificaciones técnicas & Procedimiento realizado \\
\hline & & $\begin{array}{l}\text { Promedio: Grupo A } \\
\text { de } 65.2 \pm 14.86 ; \\
\text { grupo B de } 65.28 \pm \\
13.52 ; \text { grupo } C \text { de } \\
60.08 \pm 13.42 .\end{array}$ & & $\begin{array}{l}\text { grupo A2 se les realizó estudio } \\
\text { mediante VFC para objetivar } \\
\text { resultados mediante la escala de } \\
\text { Penetración / Aspiración. }\end{array}$ \\
\hline $\begin{array}{l}\text { Lee, Lee, } \\
\text { Kang, Yi, \& } \\
\text { Kim (2016) }\end{array}$ & $\begin{array}{l}\text { Usefulness of Submental } \\
\text { Ultrasonographic } \\
\text { Evaluation for Dysphagia } \\
\text { Patients. }\end{array}$ & $\begin{array}{l}\mathrm{n}=52 \\
\text { Características: } \\
\text { pacientes con } \\
\text { trastorno de la } \\
\text { deglución. } \\
\text { Promedio: } 61.2 \\
\text { ( } \pm 16.4 \text { años). }\end{array}$ & $\begin{array}{l}\text { Equipo utilizado: Equipos } \\
\text { LOGIQ E9 (GE Healthcare, } \\
\text { Milwaukee, } \\
\text { WI, EE. UU.), con un } \\
\text { transductor curvo de 1-5 } \\
\text { MHz. } \\
\text { Ubicación transductor: Se } \\
\text { ubicó en una posición sagital } \\
\text { del área submentoniana. } \\
\text { Gel utilizado: No informa }\end{array}$ & $\begin{array}{l}\text { El examen se llevó a cabo con el } \\
\text { paciente sentado en posición vertical, } \\
\text { mientras que el cuello se mantuvo en } \\
\text { una posición neutral. } \\
\text { Los participantes debían deglutir } 5 \mathrm{ml} \\
\text { de líquido mezclado con medio de } \\
\text { contraste de la misma viscosidad que } \\
\text { el utilizado en VFC. Ambos } \\
\text { procedimientos, VFC y US se realizaban } \\
\text { de manera conjunta, para evaluar el } \\
\text { desplazamiento laríngeo y poder hacer } \\
\text { la correlación entre elevación y } \\
\text { posibilidad de penetración / } \\
\text { aspiración. }\end{array}$ \\
\hline $\begin{array}{l}\text { Li et al. } \\
\text { (2015) }\end{array}$ & $\begin{array}{l}\text { Application of B+M- } \\
\text { Mode Ultrasonography } \\
\text { in Assessing Deglutitive } \\
\text { Tongue Movements in } \\
\text { Healthy Adults. }\end{array}$ & $\begin{array}{l}\mathrm{n}=90 \\
\text { Características: } \\
\text { Sujetos sanos. } \\
\text { Promedio: Rango } \\
\text { entre 20-80 años }\end{array}$ & $\begin{array}{l}\text { Equipo utilizado: Philips IU22 } \\
\text { (Philips Medical Systems } \\
\text { Bothell, WA), máquina } \\
\text { equipada con un transductor } \\
\text { convexo C5-2. Se utilizó en } \\
\text { modo B. } \\
\text { Ubicación transductor: El } \\
\text { transductor se colocó en el } \\
\text { plano sagital submentoniano, } \\
\text { centrado entre el borde } \\
\text { anterior del hueso hioides } \\
\text { hueso y el borde posterior de } \\
\text { la sínfisis mandibular. } \\
\text { Gel utilizado: No informa. }\end{array}$ & $\begin{array}{l}\text { Los sujetos fueron examinados en } \\
\text { posición vertical con el transductor } \\
\text { fijado por un especial dispositivo para } \\
\text { evitar movimientos relativos entre } \\
\text { ellos. Los sujetos debían realizar una } \\
\text { deglución de saliva } 30 \text { segundos } \\
\text { previos a la deglución del bolo; } \\
\text { después de ese tiempo debían deglutir } \\
\text { 3-5 ml de agua mineral. Se registró en } \\
\text { video y se analizaron las siguientes } \\
\text { fases. Fase I, movimiento lingual antes } \\
\text { del inicio de la deglución (punta de la } \\
\text { lengua en contacto con dientes); Fase } \\
\text { IIA, parte media de la lengua se mueve } \\
\text { hacia arriba para contactar con el } \\
\text { paladar; Fase IIB, parte media de la } \\
\text { lengua permanece en contacto con el } \\
\text { paladar, Fase III, porción media de la } \\
\text { lengua se mueve hacia abajo y luego } \\
\text { hacia arriba para volver al estado de } \\
\text { descanso al final del proceso de } \\
\text { deglución. }\end{array}$ \\
\hline $\begin{array}{l}\text { Miura } \\
\text { et al. } \\
(2016)\end{array}$ & $\begin{array}{l}\text { Detecting pharyngeal } \\
\text { post-swallow residue by } \\
\text { ultrasound examination: } \\
\text { a case series. }\end{array}$ & $\begin{array}{l}\mathrm{n}=9 \\
\text { Características: } \\
\text { Todos presentaban } \\
\text { disfagia. } \\
\text { Promedio: } 70 \text { años. }\end{array}$ & $\begin{array}{l}\text { Equipo utilizado: Ultrasonido } \\
\text { portátil (M-Turbo; Sonosite, } \\
\text { Bothwell, WA, EE. UU.) Con } 5 \\
\text { a } 15 \mathrm{MHz} \text { (HFL50x) } \\
\text { Ubicación transductor: Se } \\
\text { ubicó el transductor } \\
\text { en el plano sagital por encima } \\
\text { del cartílago tiroides. } \\
\text { Gel utilizado: No informa. }\end{array}$ & $\begin{array}{l}\text { Se realizaron FEES y evaluación con US } \\
\text { en paralelo. Se evaluó con líquido con } \\
\text { agente colorante para mejorar la } \\
\text { visualización en el FEES. No se informa } \\
\text { sobre cantidades ni cantidad de veces } \\
\text { que los participantes debieron deglutir. } \\
\text { El residuo faríngeo se interpretó como } \\
\text { un área hiperecogénica brumosa que } \\
\text { permanecieron por encima de los } \\
\text { pliegues vocales después de la } \\
\text { deglución. }\end{array}$ \\
\hline
\end{tabular}




\begin{tabular}{|c|c|c|c|c|}
\hline Autor & Título & Muestra & Especificaciones técnicas & Procedimiento realizado \\
\hline $\begin{array}{l}\text { Miura } \\
\text { et al. } \\
\text { (2014) }\end{array}$ & $\begin{array}{l}\text { Method for detection of } \\
\text { aspiration based on B- } \\
\text { mode video } \\
\text { ultrasonography. }\end{array}$ & $\begin{array}{l}\mathrm{n}=17 \\
\text { Características: } \\
\text { Todos presentaban } \\
\text { disfagia. } \\
\text { Promedio: } 70 \text { ( } \pm 7,6 \\
\text { años). }\end{array}$ & $\begin{array}{l}\text { Equipo utilizado: Ultrasonido } \\
\text { con equipo portátil (M-Turbo; } \\
\text { Sonosite, Bothwell, WA, EE. } \\
\text { UU.) Se realizó con el uso de } \\
\text { un transductor de matriz } \\
\text { lineal de 6-15 MHz (HFL509). } \\
\text { Ubicación transductor: Se } \\
\text { ubicó el transductor a nivel de } \\
\text { cuerdas vocales. No se } \\
\text { especifica más información. } \\
\text { Gel utilizado: No informa. }\end{array}$ & $\begin{array}{l}\text { La aspiración en US se interpretó como } \\
\text { el paso de un objeto hiperecoico a } \\
\text { través de las cuerdas vocales debajo de } \\
\text { la pared anterior traqueal. Los } \\
\text { alimentos de prueba consistieron en } \\
\text { líquido fino, líquido espeso y alimento } \\
\text { sólido. Se analizaron } 42 \text { medidas en } \\
\text { total. }\end{array}$ \\
\hline $\begin{array}{l}\text { Miura } \\
\text { et al. } \\
(2018)\end{array}$ & $\begin{array}{l}\text { A Randomized } \\
\text { Controlled Trial to } \\
\text { Investigate the } \\
\text { Effectiveness of the } \\
\text { Prevention of Aspiration } \\
\text { Pneumonia Using } \\
\text { Recommendations for } \\
\text { Swallowing Care Guided } \\
\text { by Ultrasound } \\
\text { Examination. }\end{array}$ & $\begin{array}{l}\mathrm{n}=46 \\
\text { Características: } \\
\text { Usuarios con y sin } \\
\text { trastorno deglutorio. } \\
\text { Promedio: } \\
\text { Grupo intervenido } 87 \\
(82,92) \text {, grupo } \\
\text { control } 85(77,92)\end{array}$ & $\begin{array}{l}\text { Equipo utilizado: No informa } \\
\text { Ubicación transductor: No } \\
\text { informa } \\
\text { Gel utilizado: No informa }\end{array}$ & $\begin{array}{l}23 \text { participantes fueron asignados al } \\
\text { azar al grupo de intervención y } 23 \text { al } \\
\text { grupo de control. Al grupo de estudio } \\
\text { se le realizó exámenes de ultrasonido } \\
\text { durante las comidas, y se les entregó } \\
\text { recomendaciones de alimentación } \\
\text { cada } 2 \text { semanas durante un período de } \\
8 \text { semanas. Cuando se detectó residuos } \\
\text { con el US, se entregó estrategias como } \\
\text { alternar líquidos con sólidos, por } \\
\text { ejemplo. Cuando se detectó aspiración } \\
\text { con el US, se recomendó modificación } \\
\text { en la viscosidad del alimento además } \\
\text { de realizar una evaluación mediante } \\
\text { FEES para la detección de trastornos } \\
\text { estructurales. Al grupo control no se le } \\
\text { proporcionaron recomendaciones } \\
\text { sobre el tratamiento de la deglución } \\
\text { basado en exámenes de ultrasonido. }\end{array}$ \\
\hline $\begin{array}{l}\text { Morinière } \\
\text { et al. } \\
\text { (2013) }\end{array}$ & $\begin{array}{l}\text { Ultrasound analysis of } \\
\text { the upper esophageal } \\
\text { sphincter during } \\
\text { swallowing in the } \\
\text { healthy subject. }\end{array}$ & $\begin{array}{l}\mathrm{n}=25 . \\
\text { Características } \\
\text { generales: Sujetos } \\
\text { sanos. } \\
\text { Promedio: 30,11 (20 } \\
\text { - } 56 \text { años). }\end{array}$ & $\begin{array}{l}\text { Equipo utilizado: US con } \\
\text { sistema MyLabTM70 XVision } \\
\text { (Grupo Esaote, Genova, Italia) } \\
\text { y transductor tiroideo } \\
\text { Biosound LA523 ajustada a } 7 \\
\text { MHz. } \\
\text { Ubicación transductor: Se } \\
\text { ubicó el transductor en el lado } \\
\text { izquierdo del cuello para } \\
\text { obtener una vista axial a lo } \\
\text { largo del borde inferior del } \\
\text { cartílago cricoides. } \\
\text { Gel utilizado: No informa. }\end{array}$ & $\begin{array}{l}\text { Se instruyó a los participantes para que } \\
\text { deglutan de una vez } 10 \text { ml de agua a } \\
\text { temperatura ambiente entregada con } \\
\text { una jeringa. Se realizaron tres } \\
\text { grabaciones por cada uno con un lapso } \\
\text { de al menos 30s entre las grabaciones. } \\
\text { Se valoró Diámetro de apertura y } \\
\text { cierre del EES; desplazamiento anterior } \\
\text { y lateral (medidos en cm) del EES; } \\
\text { duración de la apertura de UES; y la } \\
\text { duración promedio del desplazamiento } \\
\text { del EES (medido en ms). }\end{array}$ \\
\hline $\begin{array}{l}\text { Nakamori } \\
\text { et al. } \\
(2016)\end{array}$ & $\begin{array}{l}\text { Tongue thickness } \\
\text { evaluation using } \\
\text { ultrasonography can } \\
\text { predict swallowing } \\
\text { function in amyotrophic } \\
\text { lateral sclerosis patients. }\end{array}$ & $\begin{array}{l}\mathrm{n}=36 \text { participantes } \\
\text { Características: } \\
18 \text { pacientes con ELA } \\
\text { y } 18 \text { voluntarios } \\
\text { sanos. } \\
\text { Promedio: Usuarios } \\
\text { con ELA } 62.5 \pm 9.9 \text {, } \\
\text { voluntarios sanos } \\
60.2 \pm 7.5\end{array}$ & $\begin{array}{l}\text { Equipo utilizado: Sistema SSA- } \\
\text { 770A (Toshiba, Tokio, Japón), } \\
\text { utilizando un transductor de } \\
\text { matriz convexo de 3,5 MHz. } \\
\text { Ubicación transductor: No } \\
\text { informa } \\
\text { Gel utilizado: No informa }\end{array}$ & $\begin{array}{l}\text { Los sujetos fueron examinados en una } \\
\text { posición inclinada a } 30^{\circ} \text { mientras } \\
\text { estaban sentados. El grosor de la } \\
\text { lengua se identificó como la distancia } \\
\text { entre la superficie superior e inferior } \\
\text { de los músculos linguales en el centro } \\
\text { del plano perpendicular al plano } \\
\text { horizontal de Frankfurt. } \\
\text { Posteriormente, cada participante se } \\
\text { sometió a VFC ingiriendo yogurt }\end{array}$ \\
\hline
\end{tabular}




\begin{tabular}{|c|c|c|c|c|}
\hline Autor & Título & Muestra & Especificaciones técnicas & Procedimiento realizado \\
\hline & & & & $\begin{array}{l}\text { mezclado con contraste después de la } \\
\text { confirmación de que no se había } \\
\text { producido una aspiración severa. Con } \\
\text { nueve de los pacientes con ELA se } \\
\text { repitió la evaluación con US de la } \\
\text { lengua dos o tres veces dentro de los } \\
15 \text { meses posteriores a la primera } \\
\text { evaluación. }\end{array}$ \\
\hline $\begin{array}{l}\text { Ogawa } \\
\text { et al. } \\
(2018)\end{array}$ & $\begin{array}{l}\text { Ultrasonography to } \\
\text { Measure Swallowing } \\
\text { Muscle Mass and } \\
\text { Quality in Older Patients } \\
\text { With Sarcopenic } \\
\text { Dysphagia. }\end{array}$ & $\begin{array}{l}\mathrm{n}=55 \\
\text { Características: } 14 \\
\text { participantes con } \\
\text { disfagia sarcopénica } \\
\text { posible, } 22 \text { disfagia } \\
\text { sarcopénica probable } \\
\text { y } 19 \text { sin disfagia } \\
\text { sarcopénica. } \\
\text { Promedio: } 82 \pm 7 \\
\text { años). }\end{array}$ & $\begin{array}{l}\text { Equipo utilizado: Ultrasonido } \\
\text { portátil (M-Turbo; Fujifilm } \\
\text { SonoSite, Tokio, Japón), con } \\
\text { un transductor de matriz } \\
\text { lineal y convexa de } 5 \text { a } 15 \\
\text { MHz. } \\
\text { Ubicación transductor: Se } \\
\text { ubicó el transductor en } 3 \\
\text { puntos diferentes: a un tercio } \\
\text { de la distancia desde la } \\
\text { parótida a la mandíbula para } \\
\text { el plano coronal del músculo } \\
\text { genihioideo; en la línea media } \\
\text { del piso de la boca para el } \\
\text { plano sagital del músculo } \\
\text { genihioideo, y perpendicular } \\
\text { al plano de Frankfurt para los } \\
\text { músculos de la lengua. } \\
\text { Gel utilizado: No informa. }\end{array}$ & $\begin{array}{l}\text { Los pacientes se recostaron en una } \\
\text { cama reclinable en un ángulo de } 30 \text { y } \\
\text { se mantuvieron relajados durante el } \\
\text { examen. Se examinaron el grosor y el } \\
\text { área de la lengua y los músculos } \\
\text { genihioideos (plano coronal y plano } \\
\text { sagital), realizando las mediciones en } 3 \\
\text { oportunidades para obtener la media. } \\
\text { La intensidad del eco en la región de } \\
\text { interés se expresó como valores entre } \\
0 \text { y } 256 \text { ( } 0 \text {, negro y } 256 \text {, blanco). }\end{array}$ \\
\hline $\begin{array}{l}\text { Peng, } \\
\text { Miethke, } \\
\text { Pong, \& Lin } \\
\text { (2007) }\end{array}$ & $\begin{array}{l}\text { Investigation of Tongue } \\
\text { Movements during } \\
\text { Swallowing with M- } \\
\text { Mode Ultrasonography. }\end{array}$ & $\begin{array}{l}\mathrm{n}=55 \text { ( } 30 \text { mujeres, } \\
25 \text { hombres) } \\
\text { Características: } \\
\text { Sujetos sanos } \\
\text { Promedio: } 22.3 \text { años } \\
\text { (entre } 8 \text { y } 50 \text { años). }\end{array}$ & $\begin{array}{l}\text { Equipo utilizado: Siemens } \\
\text { Sonoline SI-250 con } 3.5 \mathrm{MHz} \text {, } \\
13 \mathrm{~mm} \text { de diámetro, } \\
\text { transductor de sector } \\
\text { mecánico de } 100 \text { grados; } \\
\text { Siemens, Issaquah. } \\
\text { Ubicación transductor: Se } \\
\text { ubicó a medio camino entre el } \\
\text { borde posterior de la sínfisis y } \\
\text { el margen anterior del hueso } \\
\text { hioides en el plano medio } \\
\text { sagital. } \\
\text { Gel utilizado: Gel de } \\
\text { acoplamiento. }\end{array}$ & $\begin{array}{l}\text { Se pidió a todos los participantes que } \\
\text { deglutieran de } 3 \text { a } 5 \mathrm{ml} \text { de agua con } \\
\text { una bombilla, que esperaran } 10 \\
\text { segundos y que volvieran a deglutir sin } \\
\text { agua. Cada ciclo de deglución se repitió } \\
\text { dos veces con intervalos de al menos } \\
20 \mathrm{~s} .\end{array}$ \\
\hline $\begin{array}{l}\text { Rocha, } \\
\text { Silva, \& } \\
\text { Berti } \\
(2015)\end{array}$ & $\begin{array}{l}\text { Qualitative and } \\
\text { quantitative ultrasound } \\
\text { analysis of } \\
\text { oropharyngeal } \\
\text { swallowing. }\end{array}$ & $\begin{array}{l}\mathrm{n}=100 \\
\text { Características: } \\
\text { Adultos sanos } \\
\text { Promedio: Rango } \\
\text { entre } 20-60 \text { años. }\end{array}$ & $\begin{array}{l}\text { Equipo utilizado: Ultrasonido } \\
\text { portátil, modelo DP } 6600 \text {, } \\
\text { transductor micro-convexo. } \\
\text { Ubicación transductor: Se } \\
\text { ubicó el transductor } \\
\text { micro-convexo a 90o en la } \\
\text { región submandibular. } \\
\text { Gel utilizado: Gel conductor. } \\
\text { No detalla más información. }\end{array}$ & $\begin{array}{l}\text { Se realizaron evaluaciones ecográficas } \\
\text { de la deglución en los } 100 \text { individuos } \\
\text { utilizando consistencia líquida y } \\
\text { semisólido (yogurt). Se utilizó un } \\
\text { estabilizador en la cabeza de cada } \\
\text { participante para que el transductor se } \\
\text { pueda acoplar a 90o con la región } \\
\text { submandibular. Se consideró } \\
\text { valoración cualitativa de movilidad } \\
\text { lingual y cuantitativa de propulsión } \\
\text { lingual y desplazamiento máximo del } \\
\text { hueso hioides durante la deglución. }\end{array}$ \\
\hline
\end{tabular}




\begin{tabular}{|c|c|c|c|c|}
\hline Autor & Título & Muestra & Especificaciones técnicas & Procedimiento realizado \\
\hline $\begin{array}{l}\text { Söder \& } \\
\text { Miller } \\
(2002)\end{array}$ & $\begin{array}{l}\text { Using Ultrasound to } \\
\text { Investigate Intrapersonal } \\
\text { Variability in Durational } \\
\text { Aspects of Tongue } \\
\text { Movement During } \\
\text { Swallowing. }\end{array}$ & $\begin{array}{l}\mathrm{n}=20 \text { sujetos } \\
\text { voluntarios. } \\
\text { Características: } \\
\text { Grupo } 1 \text { sujetos con } \\
\text { disfagia neurogénica } \\
\text { con dificultades en la } \\
\text { etapa oral. Grupo 2, } \\
\text { sujetos sanos. } \\
\text { Promedio: } 54.4 \text { años } \\
\text { (rango de } 21 \text { años - } \\
79 \text { años). }\end{array}$ & $\begin{array}{l}\text { Equipo utilizado: Ultrasonido } \\
\text { LOGIQ } 500 \text { MD (GE Medical } \\
\text { Systems), con ajustes Modo B } \\
\text { y modo M. Transductor lineal } \\
546 \text { L (GE Medical Systems). } \\
\text { Ubicación transductor: Se } \\
\text { ubicó el transductor micro- } \\
\text { convexo a } 90 \text { on la región } \\
\text { submandibular. } \\
\text { Gel utilizado: Gel de contacto. } \\
\text { No detalla más información. }\end{array}$ & $\begin{array}{l}\text { Se pidió a los participantes que } \\
\text { articularan el sonido / } \mathrm{k} / \text { para obtener } \\
\text { una impresión de las condiciones } \\
\text { anatómicas. Se utilizó el transductor a } \\
\text { una profundidad de penetración de } 10 \\
\mathrm{~cm} \text { para hombres y a una profundidad } \\
\text { de penetración de } 8 \mathrm{~cm} \text { para mujeres. } \\
\text { Después de cada deglución en seco, los } \\
\text { sujetos bebieron un poco de agua y, } \\
\text { después de un descanso de } \\
\text { aproximadamente } 10 \text { segundos, } \\
\text { recibieron instrucciones de volver a } \\
\text { deglutir saliva. La investigación } \\
\text { continuó de esta manera hasta que se } \\
\text { registraron al menos } 15 \text { degluciones en } \\
\text { seco. }\end{array}$ \\
\hline $\begin{array}{l}\text { Tamburrini } \\
\text { et al. } \\
\text { (2010) }\end{array}$ & $\begin{array}{l}\text { Amyotrophic lateral } \\
\text { sclerosis: sonographic } \\
\text { evaluation of dysphagia. }\end{array}$ & $\begin{array}{l}\mathrm{n}=9 \\
\text { Características: } \\
\text { Todos presentaban } \\
\text { esclerosis lateral } \\
\text { amiotrófica (ELA). } \\
\text { Promedio: } 67 \text { años } \\
\text { (33-73). }\end{array}$ & $\begin{array}{l}\text { Equipo utilizado: Ultrasonido } \\
\text { Pro Focus System (BK } \\
\text { Medical) equipado con } \\
\text { transductor micro-convexo de } \\
\text { 5-MHz (Tipo 8803). } \\
\text { Ubicación transductor: Se } \\
\text { ubicó el transductor en el } \\
\text { cartílago tiroides. } \\
\text { Gel utilizado: No se utilizó. }\end{array}$ & $\begin{array}{l}\text { Los participantes se sometieron a } \\
\text { evaluación de US con VFC en forma } \\
\text { simultánea. Se les administró bolos de } \\
\text { líquido en } 5,10 \text { y } 15 \mathrm{ml} \text {. Se analizaron } 2 \\
\text { parámetros estáticos con VFC y } 3 \text { con } \\
\text { US; y cuatro parámetros dinámicos con } \\
\text { VFC y US. }\end{array}$ \\
\hline $\begin{array}{l}\text { Yabunaka } \\
\text { et al. } \\
(2011)\end{array}$ & $\begin{array}{l}\text { Sonographic assessment } \\
\text { of hyoid bone } \\
\text { movement during } \\
\text { swallowing: a study of } \\
\text { normal adults with } \\
\text { advancing age. }\end{array}$ & $\begin{array}{l}\mathrm{n}=30 \\
\text { Características: } \\
\text { Sujetos sanos. } \\
\text { Promedio: Rango } \\
\text { entre 20-79 años. }\end{array}$ & $\begin{array}{l}\text { Equipo utilizado: Ultrasonido } \\
\text { Xario, Toshiba Medical } \\
\text { Systems, con transductor } \\
\text { curvo (3,5-7,0 MHz) (PVT- } \\
674 \mathrm{BT}, \text { Toshiba). } \\
\text { Ubicación transductor: Se } \\
\text { ubicó el transductor de } \\
\text { manera longitudinal sobre la } \\
\text { laringe. } \\
\text { Gel utilizado: No informa. }\end{array}$ & $\begin{array}{l}\text { Los participantes fueron examinados } \\
\text { sentados en posición vertical, con la } \\
\text { espalda apoyada contra una pared } \\
\text { para controlar el movimiento. Se les } \\
\text { solicitó deglutir } 5 \mathrm{ml} \text { de agua mineral, } \\
\text { repitiendo } 5 \text { veces consecutivas el } \\
\text { proceso. Se consideró inicio y fin de } \\
\text { cada grabación el regreso de hueso } \\
\text { hioides a la posición inicial. }\end{array}$ \\
\hline $\begin{array}{l}\text { Yabunaka } \\
\text { et al. } \\
(2012)\end{array}$ & $\begin{array}{l}\text { Ultrasonographic } \\
\text { evaluation of geniohyoid } \\
\text { muscle movement } \\
\text { during swallowing: a } \\
\text { study on healthy adults } \\
\text { of various ages. }\end{array}$ & $\begin{array}{l}\mathrm{n}=60 \\
\text { Características: } \\
\text { Sujetos sanos. } \\
\text { Promedio: Rango } \\
\text { entre 29.0-67.1 años. }\end{array}$ & $\begin{array}{l}\text { Equipo utilizado: Ultrasonido } \\
\text { Xario con transductor de } \\
\text { matriz curvo (5,0 MHz) } \\
\text { (Toshiba Medical Systems, } \\
\text { Tokio, Japón). } \\
\text { Ubicación transductor: Se } \\
\text { ubicó el transductor por } \\
\text { encima de la laringe en la } \\
\text { región submentoniana. } \\
\text { Gel utilizado: No informa. }\end{array}$ & $\begin{array}{l}\text { Se utilizó el US en modo B/M para } \\
\text { evaluar el músculo genihioideo en } \\
\text { deglución. Se indicó al sujeto deglutir } \\
10 \mathrm{ml} \text { de agua. Cada paciente repitió } 5 \\
\text { veces el test. }\end{array}$ \\
\hline
\end{tabular}




\section{b) Resultados obtenidos del análisis de los artículos seleccionados}

La presente sección se divide en tres apartados: i) Valoración general del proceso deglutorio, ii) valoración de la etapa preparatoria-oral y oral del proceso deglutorio y iii) valoración de la etapa faríngea del proceso deglutorio.

\section{i) Valoración general del proceso deglutorio}

Se encontraron dos estudios que valoran de manera global el proceso deglutorio. El primero corresponde a Tamburrini et al. (2010) quienes utilizan el US para observar las características de la mecánica deglutoria, específicamente presencia de signos de alteración como escaso manejo del bolo, presencia de deglución fragmentada, movimiento lingual desorganizado. Los resultados muestran que el US presenta mayor sensibilidad para detectar precozmente signos de disfagia en la población con Esclerosis Lateral Amiotrófica (ELA) respecto a la VFC. A partir de estos resultados concluyen que el US es útil para obtener datos morfológicos y funcionales, ya que proporciona información sobre alteraciones iniciales de factores estáticos y dinámicos involucrado en la fase oral de la deglución.

El segundo es el estudio de Ogawa et al. (2018) que comparó las diferencias de masa muscular entre la disfagia sarcopénica y no sarcopénica mediante US. Se describe con precisión el área y diámetro de los músculos linguales y genihioideo, valorando las longitudes en milímetros lo que se enmarca en un manejo cuantitativo de los datos. El factor más específico para identificar la presencia de disfagia sarcopénica fue el área muscular de la lengua (sensibilidad, 0.389; especificidad, 0.947), mientras que el factor con la mayor sensibilidad fue la intensidad del brillo del área del músculo genihioideo en secciones sagitales (sensibilidad, 0.806; especificidad, 0.632).

A partir de los artículos analizados, es posible advertir que los resultados expuestos abordan tanto aspectos cualitativos (Tamburrini et al., 2010) como cuantitativos (Ogawa et al., 2018).

\section{ii) Valoración de la etapa preparatoria oral y oral del proceso deglutorio}

Seis artículos de los analizados realizan una valoración de alguna estructura participante de las etapas orales del proceso de deglución. Peng, JostBrinkmann, Miethke, \& Lin (2000) buscaron determinar si el US puede utilizarse para medir la movilidad de la lengua durante la etapa oral de la deglución. Para ello, la fase oral se dividió en cinco fases (fase I, fase de acanalimento, fase Ila, fase de transporte temprana; fase IIb, fase de transporte tardía; fase IIla, fase final temprana; y fase IIIb, fase final tardía). Sus resultados indicaron que, para la duración del movimiento durante la etapa oral, el tiempo total promedio para la población total examinada se calculó en 2,43 segundos (s), con una DS de 0,62 s. La fase IIIb fue la parte más larga (promedio, $0.91 \mathrm{~s}$ ), mientras que la fase lla fue la más corta (promedio, $0.23 \mathrm{~s}$ ). La duración más reproducible de la deglución apareció en la fase Ila (SD $=0.14 \mathrm{~s}$ ) y la duración más variable de la deglución se dio en la fase IIIb (SD = $0.47 \mathrm{~s})$. El rango promedio de movimiento total de la lengua durante la deglución en el plano medio sagital fue de $24.06 \mathrm{~mm}$ (SD = 9.99 $\mathrm{mm})$. El rango máximo se detectó en la fase lla 
(promedio, $10.73 \mathrm{~mm}$ ), mientras que se encontró que la fase Illb presentaba el rango mínimo de movimiento (promedio, $1.11 \mathrm{~mm}$ ). La parte más reproducible apareció en la fase $\mathrm{llb}(\mathrm{SD}=1,70 \mathrm{~mm}$ ). Por el contrario, la fase lla mostró la mayor fluctuación individual. Por último, la velocidad de movimiento, la velocidad durante la deglución fue de entre 2,10 $\mathrm{mm} / \mathrm{s}$ y $32,43 \mathrm{~mm} / \mathrm{s}$ (promedio, $10,34 \mathrm{~mm} / \mathrm{s}$; SD = $4,92 \mathrm{~mm} / \mathrm{s})$. Se encontró que la fase lla es la parte más rápida de la deglución (promedio, $61.81 \mathrm{~mm} / \mathrm{s}$ ), pero también fue la más variable cuando se comparó la velocidad de las cinco fases de la deglución (SD= 42.71 $\mathrm{mm} / \mathrm{s}$ ). A la inversa, la fase IIIb fue la parte más lenta y reproducible de la deglución (promedio, $3.30 \mathrm{~mm} / \mathrm{s}$; $\mathrm{SD}=3.84 \mathrm{~mm} / \mathrm{s})$. La velocidad más alta de las degluciones examinadas se calculó en 305,67 mm/s y apareció en la fase lla.

Otro estudio relacionado con la movilidad lingual es el de Hsiao et al. (2012) que buscó determinar el cambio promedio del espesor de la lengua para definir la gravedad de la disfagia en pacientes con accidente cerebrovascular (ACV), por medio de US submentoniano. Los resultados mostraron una sensibilidad y especificidad del $70.0 \%$ y $66.7 \%$, respectivamente, con respecto a la VFC. Por otra parte, en el trabajo de Söder \& Miller (2002) se estableció una duración media total de la movilidad lingual para sujetos sanos de $0.91 \mathrm{~s}$. De los diez sujetos sanos evaluados, el tiempo de movilidad más corto fue de $0,26 \mathrm{~s}$ y el más largo de 2,53 s, catalogándose de esta manera como una media no representativa al divergir considerablemente. En relación con el grupo que presentaba disfagia neurogénica con dificultades en la etapa oral, el tiempo de duración de la movilidad lingual correspondió a 2.13s (rango de 1.0 s- 3.11s.).
Rocha et al. (2015), por su parte, buscaron analizar el efecto de la edad y la consistencia del alimento de acuerdo con diversos parámetros cualitativos y cuantitativos de la deglución. Dentro de los parámetros cualitativos se describieron cinco fases: fase 1 , momento antes de recibir el bolo alimenticio con el hueso hioides en reposo; fase 2, momento de la elevación del ápice lingual para sostener el bolo alimenticio contra la región alveolar con una ligera elevación del hioides; fase 3, momento durante el cual se observa el acoplamiento de la parte medial de la lengua contra el paladar con la propulsión del bolo alimenticio; fase 4, momento de la deglución con el máximo desplazamiento del hioides y con el contacto completo de la lengua contra el paladar; y fase 5, estado de reposo de la lengua y del hueso hioides. Los parámetros cuantitativos adoptados fueron la duración de la propulsión de la lengua durante la deglución y la distancia de desplazamiento máximo del hueso hioides durante la deglución. Se determinó que todos los participantes independientemente de los grupos de edad presentaron el mismo patrón de posición de la lengua, observando además un efecto significativo en relación con la duración de la propulsión del movimiento de la lengua con respecto a la edad $(p=0.03)$, la consistencia $(p=0.001)$, así como la interacción de la consistencia con edad (F=2.05; $p=$ 0.03).

En el estudio de Li et al. (2015), se evaluó los movimientos de la lengua durante la deglución en adultos sanos utilizando la ecografía con modo $\mathrm{B}+\mathrm{M}$ (brillo más movimiento). En él observaron que en los diversos grupos de edad (20-39; 40-59: 60-80 años) los hombres tuvieron mayor desplazamiento lingual que las mujeres $(p=0.002, p<0.001, y p<0.001$, 
respectivamente). Además, detallaron que a mayor edad existe menor movilidad lingual tanto en hombres como en mujeres. No obstante, esta última observación no mostró una diferencia significativa $(p=0.116$ y $p=0.726$ ).

Finalmente, Nakamori et al. (2016) tuvieron como objetivo evaluar el grosor de la lengua mediante US como forma predictora de la función deglutoria en usuarios con ELA. Se observó clínicamente la lengua de toda la población participante, diagnosticando de esta forma a 12 de los 18 pacientes con ELA con atrofia lingual (67\%). Las lenguas de los pacientes con atrofia lingual eran más delgadas $(40.9 \pm 1.0 \mathrm{~mm})$ que las lenguas de los que no tenían atrofia lingual (incluidos los sujetos de control; 44,6 $\pm 0,7 \mathrm{~mm}, \mathrm{p}=$ $0,004)$, siendo determinados estos valores por medio del US. Se encontró que, en los pacientes con ELA, el grosor de la lengua fue significativamente menor que el de los participantes del grupo control sano $(p=$ 0.016).

Estos estudios muestran que el US permite evaluar tantos aspectos morfológicos como de la mecánica de la etapa preoral y oral de la deglución.

\section{iii) Valoración de la etapa faríngea del proceso deglutorio}

16 artículos estuvieron destinados a realizar una valoración de estructuras o acciones asociadas a la etapa faríngea (tres artículos que también habían analizado la movilidad lingual). De ellos, 11 artículos estudiaron aspectos relacionados a la movilidad del hueso hioides y/o excursión laríngea, tres sobre la posibilidad de detectar mediante US aspiración y/o penetración laríngea, uno sobre la duración del movimiento del músculo genihioideo durante la deglución, uno sobre la identificación del cierre glótico, uno sobre aspectos relacionados al tránsito faríngeo, uno sobre la movilidad de la pared faríngea lateral, y uno sobre la movilidad del EES.

Lee et al. (2016) investigaron la utilidad de la evaluación por ultrasonido en el movimiento del hueso hioides durante la deglución. Se determinó como punto de corte para detectar penetración o aspiración un desplazamiento del hueso hioides por debajo de 13,5 mm. Mientras menor era el desplazamiento del hioides mayor fue la posibilidad de penetrar o aspirar. Lo anterior presentó una sensibilidad de un $83,9 \%$ y una especificidad de un 81.0\% para detectar esta situación. En el estudio de Ahn et al. (2015) se buscó determinar en sujetos sanos, la utilidad de la evaluación por medio de US en la aproximación entre hioides-laringe al deglutir. Los datos no mostraron diferencias significativas entre género y posiciones de evaluación (sedente o supino) al momento de valorar la distancia hioides-laringe en la deglución.

Yabunaka et al. (2011) buscaron evaluar la trayectoria del movimiento del hueso hioides y los cambios relacionados con la edad, durante la deglución en sujetos sanos. Los análisis se realizaron considerando los diferentes grupos de edad: 20 - 39 años; 40-59 años; y 60 - 79 años. Se observó diferencia significativa en la duración de la deglución (tiempo total) entre el grupo de edad 20-39 y el de 40-59; y entre el de 20-39 y 60-79. (1,65 s; 1,89 s, y $1,92 \mathrm{~s}$, respectivamente; $\mathrm{p}<0.05)$. Además, se observó que la elevación máxima del hueso hioides disminuyó con la edad, encontrando diferencias significativas entre los grupos $(0.46 \mathrm{~s} ; 0,53 \mathrm{~s}$, y $0,58 \mathrm{~s}$ respectivamente, $\mathrm{p}<0.05)$. En relación con el rango de 
movimiento del músculo genihioideo durante la deglución, Yabunaka et al. (2012), observaron diferencias significativas entre hombres y mujeres en todos los rangos etarios (20-39, 40-59, 60-79 años; $p=0.001, p=0.032, p=0.013$, respectivamente). Sin embargo, no encontraron un efecto de edad ni en el grupo de hombre ni en el de mujeres $(p=0.198, p=$ 0.24 , respectivamente). En relación con la duración del movimiento del músculo genihioideo durante la deglución, nuevamente hubo diferencias estadísticamente significativas entre hombres y mujeres en cada grupo etario (20-39, 40-59, 60-79 años; $p=0.009, p=0.035, p=0.018$, respectivamente). En relación con la edad, si bien el movimiento se incrementó con los años en ambos géneros, dicho aumento no fue estadísticamente significativo (hombres $p=0.42$, mujeres $p=0.168$ ).

Huang et al. (2009) buscaron analizar la utilidad del US para la evaluación de la aproximación hioideslaringe en pacientes con accidente cerebrovascular con y sin disfagia. Se tomó como valor de corte un $40 \%$ entre la distancia laringo-hioidea durante la aproximación con la de reposo. Se obtuvo una sensibilidad de un $75.0 \%$ y una especificidad de un 77.1\%. Por su parte, Kwak et al. (2018) determinaron la influencia de la sonda nasogástrica (SNG) en la movilidad del hueso hioideo por medio del US. La media de la longitud del movimiento del hueso hioides fue de $0.81 \pm 0.36 \mathrm{~cm}$ para el grupo con SNG y de $1.14 \pm 0.36 \mathrm{~cm}$ para el mismo grupo, pero una vez retirada la SNG. Se encontró una diferencia significativa entre ambos grupos $(p=0.009)$.

Por su parte, Komori et al. (2008) realizaron un estudio con FEES, VFC, y US en forma simultánea. Determinaron que tanto VFC y US poseen una correlación significativa para valorar la distancia de la elevación laríngea $(r: 0,91 ; p<0.0001)$, y para la duración de la máxima elevación laríngea ( $r$ : 0.98; $p<$ $0.0001, n=24)$.

Kuhl et al. (2003) analizaron la excursión laríngea durante la deglución en sujetos con y sin disfagia. Se observó que, en población sana, la distancia en reposo entre hioides y tiroides fue de $220 \mathrm{~mm}$, y durante la deglución de $85 \mathrm{~mm}$ (es decir, la distancia se reduce en un 61\%). En población con disfagia, la reducción es de sólo un 42\% (185mm en reposo, y 105mm durante la deglución).

Por otra parte, Feng et al. (2015) estimaron la relación entre el tamaño y la función del músculo genihioideo y el movimiento del hueso hioides durante la deglución en adultos jóvenes sanos ubicados en tres posiciones distintas (sentado, supino, decúbito lateral derecho). Se determinó que no hubo diferencias de género en la velocidad de contracción del músculo genihioideo ( $p>0.05)$. Tampoco existió diferencias en el movimiento del hueso hioides durante la deglución entre las tres posiciones descritas $(p>0.05)$.

En el estudio de Rocha et al. (2015) se analizó el efecto de la edad y la consistencia del alimento de acuerdo con los parámetros ecográficos cualitativos y cuantitativos en la deglución orofaríngea (ya nombrados en ítem anterior). En el US cualitativo todos los participantes, independientemente de los grupos de edad, presentaron el mismo patrón de posición de la lengua (Fases 1, 2 y 5), mientras que en la fase 3 hubo una gran variabilidad entre los participantes del movimiento ondulatorio de la lengua. En la fase 4 el desplazamiento del hueso hioides aumentó como consecuencia de la 
consistencia del alimento. En el análisis del US cuantitativo, se observó un efecto significativo con respecto a la edad $(p=0.03)$, la consistencia $(p=0.00)$, así como la interacción de consistencia - edad $(\mathrm{p}=$ 0.03).

Hsiao et al. (2012) se propusieron determinar el valor de corte de la elevación laríngea para definir la gravedad de la disfagia en pacientes con accidente cerebrovascular con ultrasonografía submentoniana. Estas medidas se realizaron valorizando en milímetros el desplazamiento de estructuras con el US. Se calculó una sensibilidad de $73,3 \%$ y especificidad de $66,7 \%$ para detectar disfagias que requieren alimentación por sonda, cuyo criterio es un desplazamiento del hioides menor a 15 milímetros. Por su parte, Cheng et al. (2018) evaluaron la relación entre la contracción del músculo suprahioideo y el desplazamiento del hueso hioides en pacientes con carcinoma nasofaríngeo tratados con radioterapia por medio del US. Los valores de concordancia para el área transversal en reposo, área transversal máximo y área transversal porcentual del músculo genihioideo fue de 0.91 a $0.97,0.93$ a $0.98,0.62$ a 0.85 , y de 0.86 a $0.95 ; 0.80$ a 0.94 y 0.61 a 0.84 , respectivamente, con un intervalo de confianza de 95\%. El aumento porcentual del área de la sección transversal del músculo genihioideo se correlacionó con el desplazamiento anterior del hueso hioides $(r=0.42$, $\mathrm{p}<0.05)$, pero no con el desplazamiento superior $(r=0.27, p=0,09)$. El desplazamiento del hioides anterior y la relación de constricción faríngea se asociaron significativamente con la puntuación de la escala penetración / aspiración.

En lo relacionado a la identificación de aspiración y/o a la detección de residuos, Miura et al. (2014) observaron que el método para la detección de aspiración basado en la ecografía en modo B (modulación de brillo) presentó una sensibilidad de un $64 \%$ y una especificidad de un $84 \%$ con respecto a mediciones con VFF o FEES aplicados en forma simultánea. Otro estudio de Miura et al. (2016) detalló que el US presenta una sensibilidad de un 62\% y una especificidad de un $67 \%$ a la hora de detectar residuos faríngeos posteriores a la deglución, tras comparar los resultados ante FEES. Miura et al. (2018) valoraron la efectividad del US en la prevención de la neumonía aspirativa y evidenciaron que el US logró detectar aspiración en un 4,3\% en el grupo de estudio, al que se realizaron exámenes de ultrasonido durante las comidas. La reducción media en la frecuencia de aspiración y de generación de residuos en el grupo de estudio fue de un $31 \%$, y de un $11 \%$ en el grupo control que no recibió recomendaciones y valoraciones por medio del US.

Kim \& Kim (2012) estudiaron el movimiento de la pared faríngea lateral mediante US, encontrando que el desplazamiento en el lado débil de la faringe fue de $0.51 \mathrm{~cm}(\mathrm{DE}, 0.37)$ para los participantes con disfagia que presentaban penetración o aspiración (grupo A); de $0.94 \mathrm{~cm}$ (DE, 0.43) para los participantes con disfagia pero que no presentaban episodios de penetración ni aspiración (grupo B), y de $1.31 \mathrm{~cm}$ (DE, 0.40) para el grupo control de sujetos sanos. En relación con la duración del desplazamiento, los datos obtenidos fueron de grupo A de 2.79 s (DE, 1.88), grupo $B$ de $2.39 \mathrm{~s}(\mathrm{DE}, 1.12)$, y grupo control de $1.60 \mathrm{~s}$ (DE, 1.21) [25].

Jadcherla et al. (2006) analizó la correlación temporal del movimiento glotal entre la nasofibroscopía y el US realizado en forma 
simultánea. Se logró identificar el cierre glótico con un 99.3\% y $100 \%$ de precisión, respectivamente. La probabilidad media de perder un marco del cierre glótico a través de US fue de 0.007 (IC 95\% = 0.00080.024).

Finalmente, Morinière et al. (2013) obtuvieron mediciones morfológicas y funcionales del esfínter esofágico superior (EES) durante la deglución en sujetos sanos. Entre los datos obtenidos destaca: diámetro medio transversal del EES cerrado de 0,78 \pm $0,13 \mathrm{~cm}$, diámetro transversal interno medio del EES abierto de 0,954 $\pm 0,15 \mathrm{~cm}$, diámetro exterior transversal del EES abierto de 1,413 $\pm 0,16 \mathrm{~cm}$, grosor medio de la musculatura en abertura de 0,457 $\pm 0,16$ $\mathrm{cm}$, desplazamiento máximo anterior y lateral del EES durante la deglución de $0.42 \pm 0.12 \mathrm{~cm}$ y $0.35 \pm 0.18$ $\mathrm{cm}$, respectivamente, duración media de la apertura de $415 \pm 51,66$ ms., duración media del desplazamiento de $937 \pm 120,98$ ms., tiempo medio desde el inicio del desplazamiento de EES hasta su apertura de $330.1 \pm 80.26$ ms., y tiempo desde el cierre del EES hasta su retorno a la posición de reposo de $188.7 \pm 72.15 \mathrm{~ms}$.

Los estudios revisados en esta sección permiten concluir que el US puede complementar la evaluación tanto morfológica y funcional como la de la mecánica de la etapa faríngea.

\section{Conclusiones}

La evidencia obtenida indica que a través de US es posible valorar cualitativamente la movilidad lingual, la elevación laríngea, la movilidad faríngea lateral, la movilidad del hioides, la movilidad del músculo genihioideo, la apertura del esfínter esofágico superior y el cierre glótico.

Además, de los estudios analizados se desprende que la técnica del US ofrece posibilidades prometedoras para detectar episodios de penetración laríngea y/o aspiración.

A su vez, los resultados indican que con US se pueden obtener valoraciones cuantitativas de diversos aspectos dinámicos del proceso deglutorio como: desplazamiento lingual, dimensiones de la lengua en las diferentes fases, desplazamiento del hueso hioides, mediciones morfológicas y funcionales del EES, entre otros.

EI US es una técnica poco invasiva (Söder \& Miller, 2002) y se asocia a bajo costo, seguridad, rapidez e inocuidad (Yabunaka et al., 2011). Sin embargo, los resultados encontrados no permiten realizar una comparación con otros métodos instrumentales de evaluación ya validados en la clínica como lo son la VFC y la FEES. EI US se presenta entonces como un complemento para dichas evaluaciones, entregando información detallada y precisa que guía al clínico en el estado de la biomecánica deglutoria. Incluso en la rehabilitación podría ser útil para constatar los efectos de maniobras o ejercicios deglutorios en la musculatura.

Luego de la revisión de los artículos, es posible afirmar que existe evidencia para un futuro diseño de un protocolo fonoaudiológico a través de US. Al respecto, el estudio de Tamburrini et al. (2010) mostró la utilidad del US para obtener datos morfológicos y funcionales involucrados en la fase oral de la deglución en usuarios con ELA. Este trabajo se complementa con la investigación de Lee et al. (2016) que determinaron como punto de corte, para detectar penetración o aspiración, un desplazamiento 
del hueso hioides por debajo de 13,5 mm. Además, Morinière et al. (2013) obtuvieron mediciones morfológicas y funcionales del EES durante la deglución. Estos datos permiten pensar en una serie de indicadores que tras investigación podrían validarse como predictores de riesgo para la seguridad del proceso deglutorio.

Para la elaboración de dicho protocolo, sin embargo, aún se requiere resolver una gran cantidad de preguntas relacionadas con el procedimiento estándar de evaluación. Tal como constatamos en esta revisión, homogeneizar la técnica de ubicación del transductor en puntos anatómicos estratégicos es uno de esos cuestionamientos. También se debe resolver qué sustancia ofrece la mayor ecogenicidad al interior de la vía digestiva, cuáles son las mejores configuraciones del software del ecógrafo y las características del gel conductor. Con ello resuelto otra de las fases que queda son los estudios experimentales para comprobar la validez convergente del US, así como su eficiencia diagnóstica.

Los resultados obtenidos en esta revisión deben ser interpretados atendiendo a sus limitaciones. Una relacionada con el uso de un criterio propio de los investigadores para la elegibilidad de los artículos incluidos y otra referida a la estrategia orientada a explorar la utilidad del US en la evaluación de la deglución.

\section{Referencias}

Ahn, S. Y., Cho, K. H., Beom, J., Park, D. J., Jee, S., \& Nam, J. H. (2015). Reliability of ultrasound evaluation of hyoidlarynx approximation with positional change. Ultrasound in Medicine \& Biology, 41(5), 1221-1225. https://doi.org/10.1016/j.ultrasmedbio.2014.12.010
Cheng, D., Lee, K., Ahuja, A., \& Tong, M. (2018). Sonographic assessment of swallowing in irradiated nasopharyngeal carcinoma patients. The Laryngoscope, 128(11), 25522559. https://doi.org/10.1002/lary.27222

Chi-Fishman, G. (2005). Quantitative lingual, pharyngeal and laryngeal ultrasonography in swallowing research: $A$ technical review. Clinical Linguistics \& Phonetics, 19(6-7), 589-604. https://doi.org/10.1080/02699200500113996

Cichero, J., \& Murdoch, B. (2006). Dysphagia: Foundation, Theory and Practice. John Wiley \& Sons.

Feng, X., Cartwright, M., Walker, F., Bargoil, J., Hu, Y., \& Butler, S. (2015). Ultrasonographic evaluation of geniohyoid muscle and hyoid bone during swallowing in young adults. The Laryngoscope, 125(8), 1886-1891. https://doi.org/10.1002/lary.25224

Higgins, J., \& Green, S. (Eds.). (2011). Manual Cochrane de revisiones sistemáticas de intervenciones (v. 5.1.0). Recuperado de https://es.cochrane.org/sites/es.cochrane.org/files/public/ uploads/manual_cochrane_510_web.pdf

Hsiao, M.-Y., Chang, Y.-C., Chen, W.-S., Chang, H.-Y., \& Wang, T.-G. (2012). Application of ultrasonography in assessing oropharyngeal dysphagia in stroke patients. Ultrasound in Medicine \& Biology, 38(9), 1522-1528. https://doi.org/10.1016/j.ultrasmedbio.2012.04.017

Huang, Y.-L., Hsieh, S.-F., Chang, Y.-C., Chen, H.-C., \& Wang, T.-G. (2009). Ultrasonographic evaluation of hyoid-larynx approximation in dysphagic stroke patients. Ultrasound in Medicine \& Biology, 35(7), 1103-1108. https://doi.org/10.1016/j.ultrasmedbio.2009.02.006

Huckabee, M.-L., Macrae, P., \& Lamvik, K. (2015). Expanding Instrumental Options for Dysphagia Diagnosis and Research: Ultrasound and Manometry. Folia Phoniatrica et Logopaedica: Official Organ of the International Association of Logopedics and Phoniatrics (IALP), 67(6), 269-284. https://doi.org/10.1159/000444636

Jadcherla, S., Gupta, A., Stoner, E., Coley, B., Wiet, G., \& Shaker, R. (2006). Correlation of Glottal Closure Using Concurrent Ultrasonography and Nasolaryngoscopy in Children: A Novel Approach to Evaluate Glottal Status. Dysphagia, 21(1), 75-81. https://doi.org/10.1007/s00455005-9002-7

Kim, J.-H., \& Kim, M.-S. (2012). Lateral pharyngeal wall motion analysis using ultrasonography in stroke patients 
with dysphagia. Ultrasound in Medicine \& Biology, 38(12), 2058-2064.

https://doi.org/10.1016/j.ultrasmedbio.2012.07.028

Komori, M., Hyodo, M., \& Gyo, K. (2008). A swallowing evaluation with simultaneous videoendoscopy, ultrasonography and videofluorography in healthy controls. ORL; Journal for Oto-Rhino-Laryngology and Its Related Specialties, 70(6), 393-398. https://doi.org/10.1159/000163036

Kuhl, V., Eicke, B., Dieterich, M., \& Urban, P. (2003). Sonographic analysis of laryngeal elevation during swallowing. Journal of Neurology, 250(3), 333-337. https://doi.org/10.1007/s00415-003-1007-2

Kwak, H.-J., Kim, L., Ryu, B.-J., Kim, Y.-H., Park, S.-W., Cho, D.-G., ... Ha, K.-W. (2018). Influence of Nasogastric Tubes on Swallowing in Stroke Patients: Measuring Hyoid Bone Movement With Ultrasonography. Annals of Rehabilitation Medicine, 42(4), 551-559. https://doi.org/10.5535/arm.2018.42.4.551

Lee, Y. S., Lee, K. E., Kang, Y., Yi, T. I., \& Kim, J. S. (2016). Usefulness of Submental Ultrasonographic Evaluation for Dysphagia Patients. Annals of Rehabilitation Medicine, 40(2), 197-205. https://doi.org/10.5535/arm.2016.40.2.197

Li, C., Li, J., Zhang, C., Cao, X., Li, N., Song, D., \& Yu, T. (2015). Application of $\mathrm{B}+\mathrm{M}-$ Mode Ultrasonography in Assessing Deglutitive Tongue Movements in Healthy Adults. Medical Science Monitor: International Medical Journal of Experimental and Clinical Research, 21, 1648-1655. https://doi.org/10.12659/MSM.893591

Logemann, J. (1998). Evaluation and Treatment of Swallowing Disorders. Austin, Tex: PRO-ED.

Matsuo, K., \& Palmer, J. (2008). Anatomy and physiology of feeding and swallowing: Normal and abnormal. Physical Medicine and Rehabilitation Clinics of North America, 19(4), 691-707, vii. https://doi.org/10.1016/j.pmr.2008.06.001

Miura, Y., Nakagami, G., Yabunaka, K., Tohara, H., Hara, K., Noguchi, H., ... Sanada, H. (2016). Detecting pharyngeal post-swallow residue by ultrasound examination: A case series. Medical Ultrasonography, 18(3), 288-293. https://doi.org/10.11152/mu.2013.2066.183.yuk

Miura, Y., Nakagami, G., Yabunaka, K., Tohara, H., Murayama, R., Noguchi, H., ... Sanada, H. (2014). Method for detection of aspiration based on $\mathrm{B}$-mode video ultrasonography. Radiological Physics and Technology, 7(2), 290-295. https://doi.org/10.1007/s12194-014-0264-3

Miura, Y., Nakagami, G., Yabunaka, K., Tohara, H., Noguchi, H., Mori, T., \& Sanada, H. (2018). A Randomized Controlled Trial to Investigate the Effectiveness of the Prevention of Aspiration Pneumonia Using Recommendations for Swallowing Care Guided by Ultrasound Examination. Healthcare (Basel, Switzerland), 6(1). https://doi.org/10.3390/healthcare6010015

Morinière, S., Hammoudi, K., Marmouset, F., Bakhos, D., Beutter, P., \& Patat, F. (2013). Ultrasound analysis of the upper esophageal sphincter during swallowing in the healthy subject. European Annals of Otorhinolaryngology, Head and Neck Diseases, 130(6), 321-325. https://doi.org/10.1016/j.anorl.2012.01.008

Nakamori, M., Hosomi, N., Takaki, S., Oda, M., Hiraoka, A., Yoshikawa, M., ... Matsumoto, M. (2016). Tongue thickness evaluation using ultrasonography can predict swallowing function in amyotrophic lateral sclerosis patients. Clinical Neurophysiology: Official Journal of the International Federation of Clinical Neurophysiology, 127(2), 1669-1674. https://doi.org/10.1016/j.clinph.2015.07.032

Ogawa, N., Mori, T., Fujishima, I., Wakabayashi, H., Itoda, M., Kunieda, K., ... Ogawa, S. (2018). Ultrasonography to Measure Swallowing Muscle Mass and Quality in Older Patients With Sarcopenic Dysphagia. Journal of the American Medical Directors Association, 19(6), 516-522. https://doi.org/10.1016/j.jamda.2017.11.007

Peng, C., Jost-Brinkmann, P., Miethke, R., \& Lin, C. (2000). Ultrasonographic measurement of tongue movement during swallowing. Journal of Ultrasound in Medicine: Official Journal of the American Institute of Ultrasound in Medicine, 19(1), 15-20. https://doi.org/10.7863/jum.2000.19.1.15

Peng, C.-L., Miethke, R.-R., Pong, S.-J., \& Lin, C.-T. (2007). Investigation of tongue movements during swallowing with M-mode ultrasonography. Journal of Orofacial Orthopedics = Fortschritte Der Kieferorthopadie: Organ/Official Journal Deutsche Gesellschaft Fur Kieferorthopadie, 68(1), 17-25. https://doi.org/10.1007/s00056-007-0547-y

Rocha, S. G., da Silva, R. G., \& Berti, L. C. (2015). Qualitative and quantitative ultrasound analysis of oropharyngeal swallowing. CODAS, 27(5), 437-445. https://doi.org/10.1590/2317-1782/20152015015

Söder, N., \& Miller, N. (2002). Using ultrasound to 
investigate intrapersonal variability in durational aspects of tongue movement during swallowing. Dysphagia, 17(4), 288-297. https://doi.org/10.1007/s00455-002-0071-6

Tamburrini, S., Solazzo, A., Sagnelli, A., Del Vecchio, L., Reginelli, A., Monsorrò, M., \& Grassi, R. (2010). Amyotrophic lateral sclerosis: Sonographic evaluation of dysphagia. La Radiologia Medica, 115(5), 784-793. https://doi.org/10.1007/s11547-010-0523-2

Yabunaka, K., Konishi, H., Nakagami, G., Sanada, H., lizaka, S., Sanada, S., \& Ohue, M. (2012). Ultrasonographic evaluation of geniohyoid muscle movement during swallowing: A study on healthy adults of various ages. Radiological Physics and Technology, 5(1), 34-39. https://doi.org/10.1007/s12194-011-0132-3

Yabunaka, K., Sanada, H., Sanada, S., Konishi, H., Hashimoto, T., Yatake, H., ... Ohue, M. (2011). Sonographic assessment of hyoid bone movement during swallowing: A study of normal adults with advancing age. Radiological Physics and Technology, 4(1), 73-77. https://doi.org/10.1007/s12194010-0107-9 